\title{
Search for a Correlation Between Radio Giant Pulses and VHE Photons of the Crab Pulsar
}

\author{
N. Lewandowska, D. Elsäesser, K. Mannheim
}

\begin{abstract}
The Crab pulsar is a unique source of pulsar radio emission. Its regular pulse structure is visible over the entire electromagnetic spectrum from radio to $\mathrm{GeV}$ ranges. Among the regular pulses, radio giant pulses (GPs) are known as a special form of pulsar radio emission. Although the Crab pulsar was discovered by its GPs, their origin and emission mechanisms are currently not understood. Within the framework of this report we give a review on radio GPs and present a new idea on how to examine the characteristics of this as yet not understood kind of pulsar emission.
\end{abstract}

Keywords: neutron star, pulsar, Crab pulsar, regular pulses, giant pulses.

\section{Introduction}

Embedded in the supernova remnant SN 1054, the Crab pulsar (PSR B0531+21) is currently the only pulsar known with a pulsed emission structure seen over the entire electromagnetic spectrum. It consists of a main pulse (P1) and an interpulse (P2) occurring at the rotational phases of 70 and $\approx 110$ degrees, respectively ( [1]). Apart from these two regular pulses, further pulsed structures are visible at various frequency ranges. The precursor, for example, is an implementation which has only been observed from about 300 to $600 \mathrm{MHz}$. At higher frequencies, two additional high frequency components known as $\mathrm{HFC}$ 1 and HFC 2 are visible from $\approx 4000$ to $8000 \mathrm{MHz}$, simultaneously with a phase shift of the interpulse by about 10 degrees [1]. While the origin of P1 and P2 can be partly described by current pulsar theories, the origin of the precursor and HFC components still remains a mystery. In addition to this regular pulsed structure, the Crab pulsar is also a known source of radio GPs. Due to their properties, GPs are an unusual and exotic form of pulsar radio emission. They have been observed in a wide frequency range from $23 \mathrm{MHz}[5]$ to $15.1 \mathrm{GHz}[6,7]$ at phases of $\mathrm{P} 1$ and $\mathrm{P} 2$, and distinguish themselves by flux densities that are higher by a factor of $5 \times 10^{5}$ and pulse widths from 1-2 microseconds [8]. Several studies also confirm the occurrence of GPs at both HFC components $[9,6]$. Nevertheless none have been verified at the phase of the precursor. The emission of GPs therefore seems to be phase bounded.

Differences between GPs occurring at P1 and P2 were discovered by obervations with the Arecibo single dish telescope ${ }^{1}[11]$. These observations indicate that the time and frequency patterns of GPs at P1 are different from those at $\mathrm{P} 2$ at frequencies above $4 \mathrm{GHz}$. While Giant main pulses (GMPs) consist of narrow-band pulses of nanosecond duration, Giant interpulses (GIPs) reveal narrow emission bands with durations in the microsecond range. These differences in GPs at P1 and P2 possibly arise from different emission mechanisms underlying their development. Additionally, they contradict all previous emission theories of the Crab pulsar that assume a similar development process of both pulses.

Theoretical aspects of GPs have been broadly investigated $[15,16,4,17,18]$. Nevertheless, in spite of over 40 years since the detection of the Crab pulsar by its GPs [2], their possible origin and emission mechanism is still not understood.

The only current model based on observational data refers to GIPs above $4 \mathrm{GHz}$. The Lyutikov model [12] reconstructs the emission bands of GIPs under the assumption of higher particle density on closed magnetic field lines in contrast with the standard Goldreich-Julian model [13]. According to [14], this density is highest near the last closed magnetic field line at which a Lorentz beam develops due to magnetic reconnection events. While it moves along the closed field line, it dissipates by curvature radiation. Furthermore, the Lyutikov model also predicts the occurrence of $\gamma$-radiation together with radio GPs, and provides the motivation for simultaneous observations at $\gamma$-wavelengths.

Currently, no universal model is available for Crab radio GPs, since the Lyutikov model is only applicable to GIPs above $4 \mathrm{GHz}$, where the P2 component changes in its position by 10 degrees [1].

With apparently sporadic, short pulses of this kind, the Crab pulsar, together with its twin pulsar PSR B0540-69 in the Large Magellanic (LMC)

\footnotetext{
${ }^{1}$ http://www.naic.edu/
} 
cloud, belongs to a small group of 11 pulsars which are known to emit radio GPs. This group also consists of ordinary and millisecond pulsars (MSPs) [19], and it was thought that a common feature of them could be a high magnetic field at the light cylinder. However, no uniform accordance in all 11 pulsars could be found, and this makes the GP phenomenon a still enigmatic feature of pulsar radio emission.

\section{Multiwavelength observations}

The incentive of multiwavelength (MWL) observations is to deduce the central emission mechanism underlying the GP phenomenon. Currently it is assumed that radio GPs could be caused by coherent emission, by pair production processes or by changes in the beaming direction. To shed more light on this topic, several MWL observation campaigns were carried out. Radio GPs and $\gamma$-ray photons observed simultaneously with the Green Bank $43 \mathrm{~m}$ telescope and OSSE were examined in [10], but no correlation could be verified.

A weak correlation between radio GPs and optical photons was verified by Shearer et al. [20], who observed the Crab pulsar simultaneously with the Westerbork Synthesis Radio Telescope (WSRT) and with the TRIFFID optical photometer. They observed an increase in the optical flux by $\approx 3 \%$ during the occurrence of radio GPs, which proves that an additional non-coherent emission process accounts for the GP emission. To examine the possible MWL occurrence of GPs in a wider extent, further MWL studies at radio, optical and also $\gamma$-wavelengths are necessary in order to see if pair production processes, for example, are involved in the GP emission.

\section{$2.1 \quad$ Fermi LAT}

Arguing that the observations in [10] were based on insufficient sensitivity, several observation campaigns of the Crab pulsar were carried out with the GBT and Fermi $\mathrm{LAT}^{2}$ to examine the assumptions of the Lyutikov model [21-23]. With a collection area of $0.8 \mathrm{~m}^{2}$, a total of 77 Fermi photons were detected in more than 10 hours of observations in the energy range between $100 \mathrm{MeV}$ and $5 \mathrm{GeV}$, simultaneously with over 210000 radio GPs at a frequency of $8.9 \mathrm{GHz}$ (Figure 1) $[22,23]$. In each case, a search was made for a correlation between the GP rate and single Fermi photons in addition to a change in the $\gamma$-ray flux around single GPs. However, Bilous et al. conclude that with $95 \%$ probability the energy flux in a $30 \mathrm{~ms}$ time window is not higher than 6 times the average flux, which suggests that coherent emission is the responsible mechanism for GIPs (Giant interpulses, see Introduction). One of their conclusions refers to the possible existence of a correlation at very high energies $\geq 100 \mathrm{GeV}$, at which a decisive number of photons for a correlation analysis cannot be provided in a reasonable time span by Fermi LAT [21].

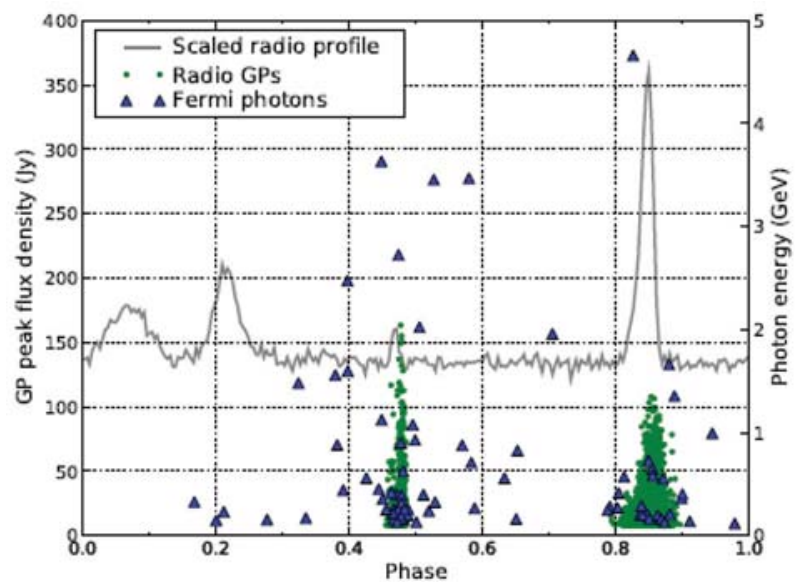

Fig. 1: Distribution of GP peak flux density and energy of $\gamma$-ray photons detected by Fermi over the pulsar rotational phase [Bilous et al.(2010)]

\subsection{Cherenkov telescopes}

One key question resulting from the observations with Fermi LAT is whether the correlation does not exist at higher energies. At this point, Imaging Air Cherenkov Telescopes (IACTs) with a general sensitivity $>60 \mathrm{GeV}$ are essential. Telescopes of this kind observe $\gamma$-rays indirectly through the detection of extensive air showers. When a $\gamma$-ray reaches the atmosphere of the Earth, it strikes one of its molecules and produces a cascade of secondary particles. These secondary particles produce Cherenkov radiation moving nearly at the speed of light at a height of about $10-20 \mathrm{~km}$ in the atmosphere. The Cherenkov light is emitted in the form of a cone around the direction of the primary particle (Figure 2). These brief flashes of Cherenkov radiation are imaged by IACTs.

With a primary mirror $17 \mathrm{~m}$ in diameter in each case, Major Atmospheric Gamma Imaging Cherenkov (MAGIC) ${ }^{3}$ telescopes are currently the biggest IACTs worldwide. Among other IACTs, for example CANGAROO III, H.E.S.S. and VERITAS, the MAGIC telescopes were the first to detect the pulsed emission of the Crab pulsar at an energy threshold of $25 \mathrm{GeV}$ provided by a special trigger system [24]. Their large mirrors enable the detection of single VHE photons on short time scales. Due to the short widths of radio GPs, an accurate timing system

\footnotetext{
${ }^{2}$ http://www-glast.stanford.edu/

${ }^{3}$ http://magic.mppmu.mpg.de/
} 
down to at least microseconds is needed for a correlation analysis with VHE photons. Employing the Global Positioning System, MAGIC provides a time stamp with an accuracy of $200 \mathrm{~ns}$ for each single VHE photon, and permits the user to decide whether it arose simultaneously with a radio GP. Thus MAGIC affords a unique opportunity to search for VHE photons coinciding with GPs at a sensitivity exceeding previous studies using e.g. Fermi LAT, and to test various models dealing with the possible generation of Crab GPs $[15,16,4,17,18,12]$.

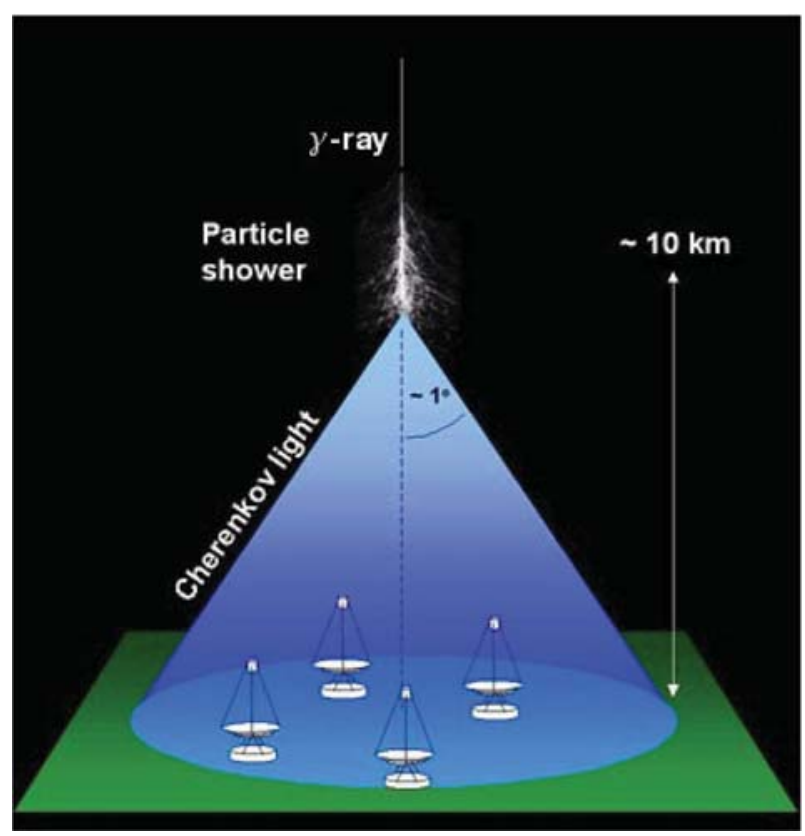

Fig. 2: Illustration of the air shower technique http://icc.ub.edu/gp_oa.php

\section{References}

[1] Moffett, D. A., Hankins, T. H.: Astrophysical Journal, 1996, Vol. 468, p. 779.

[2] Staelin, D. H., Reifenstein, E. C.: III, Science, 1968, Vol. 162, (3861), p. 1481-1483.

[3] Soglasnov, V.: Proceedings of the 363rd WEHeraeus Seminar on Neutron Stars and Pulsars 40 years after the discovery, 2007, MPE-Report 291, p. 68 .

[4] Hankins, T. H.: Nature, 2003, Vol. 422, (6928), p. $141-143$.

[5] Popov, M. V., Kuzmin, A. D., Ulyanov, O. M., Deshpande, A. A., Ershov, A. A., Kondratiev, V. I., Kostyuk, S. V., Losovsky, B. Ya., Soglasnov, V. A., Zakharenko, V. V.: On the Present and Future of Pulsar Astronomy, 26th meeting of the IAU, Joint Discussion 2, 2006.
[6] Hankins, T. H.: Proceedings of the 17rth Colloquium of the IAU held in Bonn, 2000, Vol. 202, p. 165.

[7] Jessner, A., Popov, M. V., Kondratiev, V. I., Kovalev, Y. Y., Graham, D., Zensus, A., Soglasnov, V. A., Bilous, A. V., Moshkina, O. A.: Astronomy and Astrophysics, 2010, Vol. 524, (id.A60).

[8] Kuzmin, A. D.: Astrophysics and Space Science, 2007, Vol. 308, 1-4, p. 563-56.

[9] Jessner, A., Slowikowska, A., Klein, B., Lesch, H., Jaroschek, C. H., Kanbach, G., Hankins, T. H.: Advances in Space Research, 2005, Vol. 35, 6, p. 1166-1171.

[10] Lundgren, S. C., Cordes, J. M., Ulmer, M., Matz, S. M., Lomatch, S., Foster, R. S., Hankins, T.: Astrophysical Journal, 1995, Vol. 435, 6928 , p. 433.

[11] Hankins, T. H., Eilek, J. A.: Astrophysical Journal, 2007, Vol. 670, 1, p. 693-701.

[12] Lyutikov, M.: Monthly Notices of the Royal Astronomical Society, 2007, Vol. 381, 3, p. $1190-1196$.

[13] Goldreich, J., Julian, W. H.: Astrophysical Journal, 1969, Vol. 157, p. 86.

[14] Gruzinov, A.: Physical Review Letters, 2005, Vol. 94, 2, id. 021101.

[15] Mikhailovskii, A. B., Onishchenko, O. G., Smolyakov, A. I.: Soviet Astr. Lett. (Tr: Pisma), 1985, Vol. 11, No. 2/MAR/APR, p. 78.

[16] Weatherall, J. C.: Astrophysical Journal, 2001, Vol. 559, 1, p. 196-200.

[17] Petrova, S. A.: Astronomy and Astrophysics, 2004, Vol. 424, p. 227-236.

[18] Istomin, Y. N.: Astronomical Society of the Pacific, 2004, p. 369.

[19] Slowikowska, A., Jessner, A., Kanbach, G., Klein, B.: Proceedings of the 363rd WE-Heraeus Seminar on Neutron Stars and Pulsars 40 years after the discovery, 2007, MPE-Report 291, p. 64 .

[20] Shearer, A., Stappers, B., O'Connor, P., Golden, A., Strom, R., Redfern, M., Ryan, O.: Science, 2003, Vol. 301, 5632, p. 493-495.

[21] Bilous, A. V., Kondratiev, V. I., McLaughlin, M. A., Mickaliger, M., Lorimer, D. R., Ransom, S. M., Lyutikov, M., Stappers, B., Langston, G. I.: 2009 Fermi Symposium, eConf Proceedings C091122. 
[22] Bilous, A. V., Kondratiev, V. I., McLaughlin, M. A., Ransom, S. M., Lyutikov, M., Mickaliger, M., Stappers, B., Langston, G. I.: Proceedings of the ISKAF2010 Science Meeting. June 10-14, 2010.

[23] Bilous, A. V., Kondratiev, V. I., McLaughlin, M. A., Ransom, S. M., Lyutikov, M., Mickaliger, M., Langston, G. I.: Astrophysical Journal, 2011, Vol. 728, 110.
[24] Aliu, E., et al.: Science, 2008, Vol. 322, 5905, p. 1221.

Natalia Lewandowska

Dominik Elsäesser

Karl Mannheim

University of Würzburg 